\title{
Estudo comparativo das microdeformações resultantes de cargas estáticas axiais e não axiais em próteses de três elementos implantossuportadas
}

\author{
Comparative study of micro deformations resulting from axial and non-axial static loads \\ under three prosthetic implant elements \\ Júlio Ferraz CAMPOSa, Vinícius Anéas RODRIGUESª, Ivan BALDUCCI ${ }^{a}$, \\ Gabriela Nogueira de Melo NISHIOKA ${ }^{\mathrm{b}}$, Renato Sussumu NISHIOKA ${ }^{\mathrm{a}}$
}

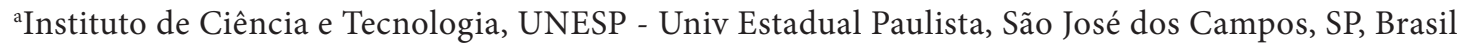

Faculdade de Odontologia, UNESP - Univ Estadual Paulista, Araraquara, SP, Brasil

\begin{abstract}
Resumo
Introdução: O controle das microdeformações ao redor dos implantes é um fator que pode levar a uma melhor osseointegração e manutenção das estruturas ósseas. Objetivo: Avaliar a distribuição de tensões ao redor dos implantes, utilizando-se a extensometria, sob a influência da aplicação de cargas estáticas axiais e não axiais em próteses parciais fixas de três elementos, em diferentes pontos de aplicação de carga. Material e método: Em um bloco de poliuretano, foram instalados três implantes de hexágono interno e, sobre esses implantes, pilares protéticos microunit foram conectados com torque de $20 \mathrm{Ncm}$. Cilindros plásticos foram utilizados para realização dos enceramentos padronizados que, posteriormente, foram fundidos em liga de cobalto cromo. Extensômetros foram colados na superfície do bloco ao redor dos três implantes. As estruturas metálicas foram parafusadas com torque de $10 \mathrm{Ncm}$ cada. Em seguida, foram realizados carregamentos verticais estáticos de $30 \mathrm{kgf}$, durante dez segundos, em cinco pontos pré-determinados (A, B, C, D e E), utilizando um dispositivo de aplicação de cargas (DAC). Resultado: Os dados foram analisados pelo teste RM ANOVA, que indicou que o efeito entre o ponto de aplicação de carga e os diferentes corpos de prova não foi estatisticamente significante, enquanto que, para o efeito entre os pontos de aplicação de carga, houve diferença $(\mathrm{p}=0,0001)$. Em seguida, aplicou-se o teste de comparação múltipla de Tukey. Conclusão: A aplicação de carga sobre os pontos não axiais D e E produziu um aumento da magnitude de microdeformação ao redor dos implantes.
\end{abstract}

Descritores: Implantes dentários; prótese parcial fixa; prótese dentária.

\begin{abstract}
Introduction: The control of the micro strain surrounding implants is a factor that can lead to a better osseointegration and maintenance of bone structures. Objective: evaluate in vitro, using strain gauge analysis, the influence of the axial and non-axial static load in implant-supported fixed partial dentures, varying the point of load application. Material and method: In a block of polyurethane three internal hexagonal implants were installed, on implants abutments microunit were connected with torque of $20 \mathrm{Ncm}$, and plastic prosthetic cylinders were screwed on to the abutments, which received standard patterns cast in Co-Cr alloy $(n=10)$. Four strain gauges were bonded on the surface of the block tangentially to the three implants. Each metallic structure was screwed onto the microunit with a $10 \mathrm{Ncm}$ torque. With a load application device (DAC) static vertical loads of 30kgf were applied for ten seconds at five predetermined points (A, B, C, D and E). Result: Result analyzed by RM ANOVA, in which the effect of the point of load application and differents specimens were not statistically significant, whereas regarding the points of load application presented statistical differenced $(\mathrm{p}=0.0001)$. Applying Tukey's multiple comparison tests. Conclusion: The application of load on the non-axial points $\mathrm{D}$ and $\mathrm{E}$ produced an increase in the magnitude of micro strain around implants.
\end{abstract}

Descriptors: Dental implants; denture, partial, fixed; dental prosthesis 


\section{INTRODUÇÃO}

A utilização de implantes osseointegrados em próteses múltiplas apresenta bons resultados clínicos, permitindo uma previsibilidade do sucesso $^{1}$. A previsibilidade do protocolo original de Bränemark incentivou o desenvolvimento de técnicas focadas nas reabilitações de pacientes parcialmente desdentados, o que levou ao crescente progresso da Implantodontia; dessa forma, diversos estudos têm confirmado a confiabilidade, a preferência e a satisfação quanto ao método de reabilitação oral ${ }^{2,3}$. Porém, a grande utilização dessa metodologia criou a necessidade de estudar fatores que levam à falha de implantes e prótese nessa técnica. A sobrecarga oclusal é um dos fatores que levam à perda óssea peri-implantar. Estudos realizados por meio de uma análise clínica retrospectiva sobre a relação entre a fratura de implantes e a sobrecarga oclusal observaram que, em grande maioria das vezes, as fraturas ocorrem em regiões posteriores associadas à presença de e disfunções, que levam a uma sobrecarga oclusal. Note-se que o controle das cargas oclusais é importante para a manutenção dos tecidos ao redor dos implantes ${ }^{4,5}$.

O controle das deformações envolve diferentes fatores biológicos e mecânicos, sendo alguns desses fatores de controle do profissional, como a escolha e a utilização de componentes protéticos, sendo a configuração geométrica da plataforma do implante com o pilar protético importante na distribuição das microdeformações na interface osso/implante ${ }^{6}$. As forças aplicadas sobre a prótese são posteriormente transmitidas para a interface osso/implante ${ }^{7}$. O controle das cargas biomecânicas influencia na manutenção da estrutura óssea, pois as forças oclusais afetam o osso adjacente ao implante. Fisiologicamente, quando o osso é submetido a cargas mecânicas, este se adapta a essa situação, caracterizada pela remodelação óssea. O estímulo mecânico dentro de um limiar de carga pode resultar no desenvolvimento ósseo devido ao aumento da sua densidade e aposição. Porém, quando esse estímulo mecânico ultrapassa um limiar aceitável, resulta em microdanos por fadiga e, consequentemente, gera reabsorção óssea ${ }^{4,8}$.

O conhecimento das distribuições dessas cargas permite um melhor planejamento clínico. Diferentes estudos in vitro, utilizando-se a técnica de extensometria e o poliuretano para simulação da estrutura óssea, verificaram o comportamento biomecânico em diferentes posições dos implantes e também em diferentes componentes para prótese sobre implantes ${ }^{8-10}$. A extensometria linear elétrica é uma proposta concreta para avaliar a distribuição de cargas estáticas ao redor de implantes ${ }^{10-12}$.

São muitas as variáveis a serem pesquisadas a respeito dos componentes, da localização dos implantes no osso e dos pontos de aplicação de carga mais favoráveis. No entanto, este estudo, por meio de uma avaliação in vitro, visa a analisar as distribuições de tensões ao redor de implantes de hexágono interno, utilizando componentes micronut e simulando uma estrutura metálica de prótese fixa de três elementos, comparando a aplicação de carga em pontos axiais e não axiais.

\section{MATERIAL E MÉTODO}

\section{Confecção do bloco de poliuretano}

Uma matriz metálica foi utilizada para confecção do bloco de poliuretano ${ }^{13}$. A matriz de alumínio é constituída por uma placa horizontal, duas placas verticais maiores, duas placas verticais menores e oito parafusos, que possibilitaram a fixação das placas: a) placa horizontal: base retangular exibindo $111 \mathrm{~mm}$ de comprimento por $61 \mathrm{~mm}$ de largura e $6 \mathrm{~mm}$ de espessura; b) placa vertical maior: possui formato retangular com dimensões de $95 \mathrm{~mm}$ de comprimento por $30 \mathrm{~mm}$ de largura e $8 \mathrm{~mm}$ de espessura, e c) placa vertical menor: estrutura retangular apresentando $61 \mathrm{~mm}$ de comprimento por $30 \mathrm{~mm}$ de largura e 8 $\mathrm{mm}$ de espessura.

A matriz de alumínio foi montada por meio da união da placa horizontal às placas verticais e do uso dos parafusos, formando um recipiente retangular com dimensões internas de $95 \times 45 \times$ $30 \mathrm{~mm}$.

Em seguida, uma resina de poliuretano (Poliuretano F16 Axson, Cercy - France), constituída por uma base e um catalisador, foi proporcionada e misturada até uma homogeneização total dos componentes. A resina foi vertida na matriz de alumínio e foram aguardados 20 minutos para que ocorresse a polimerização final do poliuretano.

\section{Inserção dos implantes e conexão dos pilares protéticos}

Para o correto posicionamento dos implantes no bloco de poliuretano, foi utilizado um conjunto de matrizes de alumínio, simulando uma situação clínica de posicionamento linear (Figura 1).

A partir da fixação da matriz no bloco de poliuretano (Figura 1B), padronizaram-se a distância e os locais para a inserção dos três implantes no bloco de poliuretano. Para a realização das perfurações, orientou-se por cilindros coloridos, com diâmetros progressivamente maiores: branco- 2,0 $\mathrm{mm}$; amarelo- $3,0 \mathrm{~mm}$ e azul- 3,15 mm. Para as perfurações, foi utilizado o conjunto de fresas padronizadas e convencionais para cirurgias: broca piloto; 2,0; 3,0 e 3,15 mm (AS Technology titaniumfix), sendo acopladas ao contra-ângulo Koncept Kavo Ind. Com. Ltda., Chapecó Brasil) com redução 20:1, conectado ao motor elétrico (MC 101 Omega, Dentsclar - Brasil).

Foram utilizados três implantes com 3,75 mm de diâmetro por $13 \mathrm{~mm}$ de comprimento e plataforma de assentamento de 4,1 mm com hexágono interno (AS Technology titaniumfix). A velocidade média utilizada foi de 14 rotações por minuto, com torque ajustado de $40 \mathrm{Ncm}$. O posicionamento final dos implantes pode ser observado no esquema da Figura 1C.

A seguir, conexões protéticas do tipo Microunit (AS Technology titaniumfix), com cinta metálica de $2 \mathrm{~mm}$, foram instaladas sobre os implantes com torque de $20 \mathrm{Ncm}$, com auxílio de um torquímetro mecânico progressivo (AS Technology titaniumfix) (Figura 1D) 

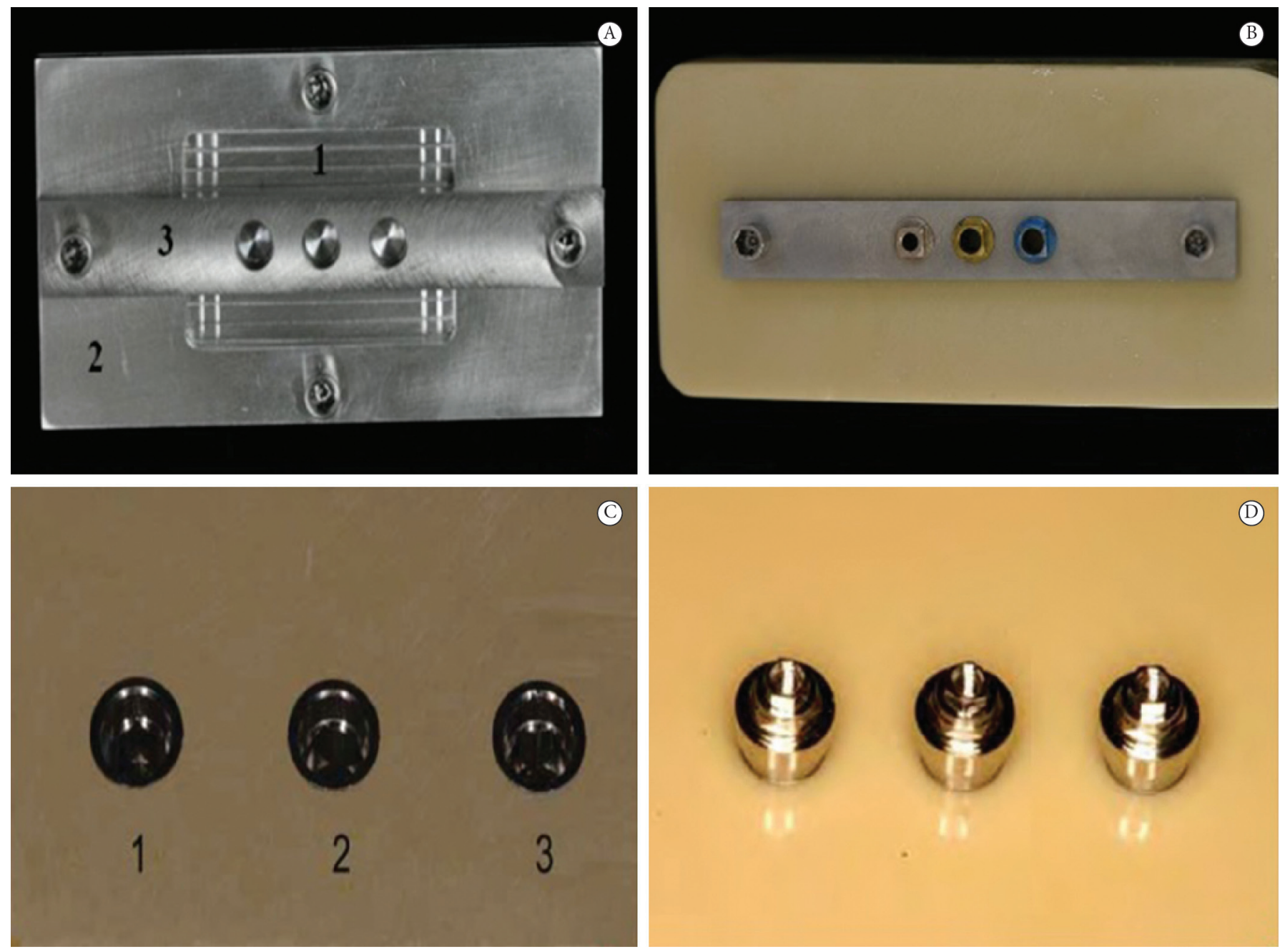

Figura 1. A) Matriz montada: para a padronização do enceramento e base para a realização das perfurações. B) Matriz fixada por parafusos verticais no bloco de poliuretano e a apresentação em posição dos anéis rosqueáveis, para orientação das perfurações. C) Implantes posicionados no bloco de poliuretano. D) Pilares protéticos Microunit conectados nos implantes com torque de $20 \mathrm{Ncm}$

\section{Obtenção dos corpos de prova}

\section{Enceramento inclusão e fundição}

Foram realizados dez enceramentos padronizados, obtidos com o auxílio de uma matriz metálica, sendo posicionados sobre as coifas plásticas (AS Technology titaniumfix) e unidos através de cera liquefeita.

Cada enceramento foi unido a um conduto de alimentação, fixado na base formadora do cadinho (DCL Dental Campinense Ltda, São Paulo - Brasil); após a inserção e a cristalização do revestimento, foram removidos da base formadora de cadinho e do anel de silicone. O bloco de revestimento foi levado ao forno (EDG 1800 EDG - Brasil) para aquecimento e volatilização da cera e do plástico. Utilizou-se o seguinte ciclo: para o préaquecimento, temperatura de $800{ }^{\circ} \mathrm{C}$ com patamar de 30 minutos, seguido da elevação da temperatura a $900{ }^{\circ} \mathrm{C}$ com patamar de 30 minutos.

Após a fundição, foi utilizado um martelete de pneumático, sendo que o excesso de revestimento foi removido com uma tesoura reta para ouro (SS White - Brasil). Posteriormente, foi realizado jateamento (Easyblast Bego, Bremen - Alemanha). As estruturas metálicas foram assentadas no bloco de poliuretano e a adaptação das estruturas, verificada sem realização do aperto dos parafusos. Cada estrutura metálica ou corpo de prova foi numerado e codificado com o grupo correspondente, obtendo-se um total de dez amostras.

\section{Extensometria}

Quatro extensômetros lineares elétricos (KFG-02-120-c111N30C2 Kyowa Eletronic Instruments Co, Ltd - Tokyo - Japan) foram colados na superfície superior do bloco de poliuretano, tangenciando a plataforma dos implantes. Previamente, a superfície do bloco foi polida com lixa d'água de granulação 220 , seguida da limpeza com álcool isopropílico.

Utilizou-se uma pequena quantidade de adesivo à base de cianoacrilato (Super Bonder Loctite, São Paulo - Brasil) para colagem dos extensômetros. Previamente aos procedimentos de colagem, foi realizada a aferição de cada extensômetro, para verificar o valor de resistência elétrica, que corresponde a $120 \Omega$, utilizando-se aparelho multímetro (Minida ET 2055: Minida São Paulo - Brasil). Foram coladas placas terminais na superfície superior do bloco, nas quais foram adaptadas as 
conexões elétricas. Para registrar adequadamente os valores de alteração de resistência gerados pela deformação do bloco de poliuretano, os valores de resistência elétrica foram amplificados por um aparelho condicionador (Model 5100 Scanner - System 5000 - Instruments Division Measurements Group, Inc., Raleigh, North Carolina - EUA), que também realizou sua conversão de analógico para digital.

As variações elétricas foram aritmeticamente transformadas para unidades de microdeformação $(\mathrm{m} \varepsilon)$ pelo software Strain-Smart de aquisição de dados, responsável pelo registro das informações, tais como interpretações, modificações e processamentos dos sinais.

\section{Aplicação da carga vertical estática}

Foi utilizado um dispositivo de aplicação de carga para realização dos carregamentos estáticos verticais de diversas magnitudes sobre os corpos de prova.

Após o posicionamento de cada corpo de prova sobre os pilares protéticos, foram realizados os ajustes dos parafusos de retenção com auxílio de uma chave de ponta hexagonal de 1,2 mm (AS Technology titaniumfix), com torque aproximado de 10 $\mathrm{Ncm}$.

Foram selecionados cinco pontos de aplicação de carga axial: o ponto A, localizado sobre orifício do parafuso de retenção do implante 1; o ponto B, localizado sobre o orifício do parafuso de retenção do implante 2; o ponto $\mathrm{C}$, localizado sobre o orifício do parafuso de retenção do implante 3 ; o ponto $\mathrm{D}$, localizado a 4 $\mathrm{mm}$, e o ponto E, a $5 \mathrm{~mm}$ do ponto C, conforme a Figura 2.

A carga estática vertical utilizada foi de $30 \mathrm{kgf}^{14}$ durante dez segundos, com o auxílio do dispositivo de aplicação de carga. No delineamento deste experimento, foi seguido um esquema fatorial tipo $1 \times 5$, sendo que as variáveis dependentes estudadas foram os pontos de aplicação de carga axiais $\mathrm{A}, \mathrm{B}$ e $\mathrm{C}$, e não axiais D e E. A variável independente foi a microdeformação $(\mathrm{m} \varepsilon)$ obtida na extensometria, durante a aplicação de carga. Foram realizadas três aplicações em cada ponto, um total de 150

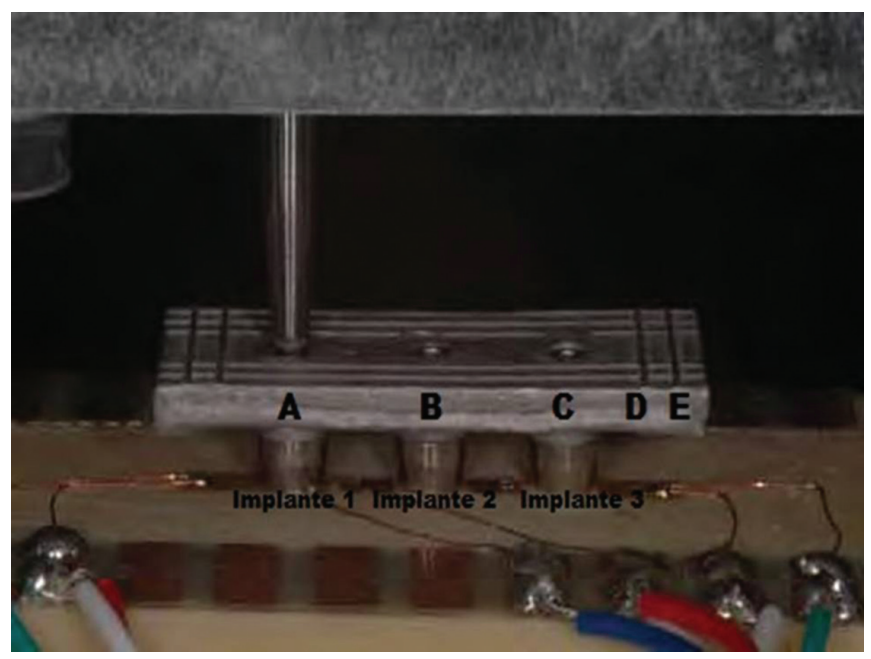

Figura 2. Pontos de aplicação de cargas axiais (A, B e C) e não axiais (D e E) medições, gerando um valor de média para cada ponto, sendo três médias axiais $(\mathrm{A}, \mathrm{B}, \mathrm{C})$ e dois não axiais $(\mathrm{D}, \mathrm{E})$ por corpo de prova, com um total de dez corpos de prova. Assim, obtivemos 50 resultados, em média, dos diferentes pontos de aplicação, em valores de microdeformação.

O sistema formado pela estrutura metálica apoiada sobre três pilares micronut constitui a unidade experimental. Os corpos de prova foram divididos aleatoriamente, estabelecidos pelas cinco variáveis experimentais.

A estatística descritiva constituiu-se no cálculo de médias e desvios padrão, e representação gráfica.

Os dados foram submetidos à análise de variância (ANOVA) de um fator de efeito fixo (ponto de aplicação). Após o teste ANOVA, seguiu-se a aplicação do teste de comparação Múltipla de Tukey para os fatores que mostraram diferença estatisticamente significante, adotando-se nível de significância de 5\%.

\section{RESULTADO}

Foram utilizados os valores de microdeformação em módulo, permitindo comparações entre as magnitudes de deformação resultante do ponto de aplicação de carga. Inicialmente, foram obtidas as médias de microdeformação nos quatro extensômetros (SG) e nos cinco pontos de aplicação de carga, demonstradas na Figura 3.

Analisando-se as Figuras acima, observou-se que as maiores microdeformações ocorreram nos extensômetros localizados próximos aos pontos de aplicação de carga $\mathrm{D}$ e E, que são os pontos não axiais. Enquanto que, para os extensometros localizados próximos aos pontos de aplicação de carga A, B e $\mathrm{C}$, que correspondem aos pontos axiais, ocorreu uma menor microdeformação. Em seguida, foi obtida a média dos quatro extensômetros e realizada a estatística descritiva dos cinco pontos de aplicação de carga, que estão representados na Tabela 1.

Os dados obtidos em microdeformação para as variáveis de ponto de aplicação de carga foram submetidos ao teste estatístico RM ANOVA de um fator, e os resultados estão apresentados na Tabela 2.

Observou-se, por meio da Tabela 2, teste RM ANOVA, que o efeito entre o ponto de aplicação de carga e corpo de prova não foi estatisticamente significante, enquanto que, para o efeito ponto de aplicação de carga, houve diferença estatisticamente significante $(\mathrm{p}=0,0001)$. Em seguida, aplicou-se o teste de comparação múltipla de Tukey, cujos dados obtidos estão representados na Tabela 3.

\section{DISCUSSÃO}

Neste estudo, foram selecionados cinco pontos para aplicação de carga, sendo três pontos para carga axial A, B e C, localizados sobre o orifício dos parafusos de retenção do implante 1, 2 e 3, respectivamente, e mais dois pontos, para aplicação de carga não axiais, $\mathrm{D}$ e E, localizados a $4 \mathrm{~mm}$ e $5 \mathrm{~mm}$ do ponto C, respectivamente. Com a aplicação da carga, seja esta 
Gráfico de colunas com valores médios de microdeformação $(\mu \varepsilon)$ obtidos em cada extensômetro em relação ao ponto de aplicação de carga.



Figura 3.Valores das médias dos pontos em cada extensômetro

Tabela 1. Dados de microdeformação referentes à variável do ponto de aplicação de carga, obtidos a partir da média dos quatro extensômetros

\begin{tabular}{ccccc}
\hline $\begin{array}{c}\text { Ponto de } \\
\text { Aplicação }\end{array}$ & N $^{*}$ & Média & $\begin{array}{c}\text { Desvio } \\
\text { Padrão }\end{array}$ & $\begin{array}{c}\text { Coeficiente } \\
\text { de Variação }\end{array}$ \\
\hline A & 10 & 228,08 & 24,46 & 10,72 \\
B & 10 & 254,84 & 29,63 & 11,63 \\
C & 10 & 270,78 & 23,42 & 8,65 \\
D & 10 & 304,00 & 44,50 & 14,66 \\
E & 10 & 349,60 & 11,90 & 290,1 \\
\hline
\end{tabular}

*Número de corpos de prova.

Tabela 2. Resultados do teste RM ANOVA

\begin{tabular}{cccccc}
\hline Efeito & gl & SQ & QM & F & P \\
\hline $\begin{array}{c}\text { Ponto de Apli- } \\
\text { cacão (PA) }\end{array}$ & 4 & 88244.1 & 22061 & 23.73 & $0.001^{*}$ \\
\hline Interação (C/PA) & 9 & 18190.9 & 2021.2 & 2.17 & $0.048^{*}$ \\
\hline Resíduo II & 36 & 33473.6 & 929.8 & & \\
Total & 49 & 139908.6 & & & \\
\hline
\end{tabular}

Tabela 3. Teste de Tukey para as médias de microdeformação nas cinco condições experimentais (pontos de aplicação de carga)

\begin{tabular}{ccc}
\hline $\begin{array}{c}\text { Ponto de Aplica- } \\
\text { ção }\end{array}$ & Média & $\begin{array}{c}\text { Grupos } \\
\text { Homogêneos }\end{array}$ \\
\hline E & 349,6 & $\mathrm{~A}$ \\
D & 304 & $\mathrm{~B}$ \\
C & 270,78 & $\mathrm{BC}^{*}$ \\
B & 254,84 & $\mathrm{CD}^{*}$ \\
A & 228,08 & $\mathrm{D}$ \\
\hline
\end{tabular}

${ }^{*}$ Médias seguidas de mesma letra não diferem estatisticamente. localizada no ponto A, B, C, D ou E, verificou-se que as maiores microdeformações ocorreram nos extensômetros localizados próximos ao ponto de aplicação de carga, o que indica que as tensões ocorridas ao redor dos implantes dependem do local dessa aplicação.

Observou-se também um aumento nos valores de microdeformação quando o carregamento é feito sobre os pontos D e E. A aplicação de carga sobre o ponto E gerou valores estatisticamente maiores do que os pontos de aplicação axial (A, B e C). Estes resultados sugerem que houve um aumento das tensões ao redor dos implantes à medida que o contato oclusal se afastou do centro do implante 3, criando uma situação de cantilever $^{15}$. Assim, o aumento da distância da aplicação da carga em relação ao centro do implante aumenta o braço de alavanca, gerando mais tensões ao redor dos implantes ${ }^{12}$.

Normalmente, em estudos biomecânicos de Implantodontia, as cargas utilizadas nos carregamentos são em torno de 35 a 100 $\mathrm{N}^{16-18}$; porém, em 1995, Mericske-Stern et al. ${ }^{14}$ investigaram a força oclusal de pacientes portadores de prótese parcial fixa sobre implantes e encontraram valores médios de máxima força oclusal de $206,1 \pm 87,6 \mathrm{~N}$ para primeiros pré-molares, de 209,8 $\pm 88,2$ $\mathrm{N}$ para molares e de $293,2 \pm 98.3 \mathrm{~N}$ para segundos pré-molares. Neste estudo, foi utilizada uma carga de $30 \mathrm{kgf}$, equivalente a aproximadamente $294 \mathrm{~N}$, sendo aplicada através do dispositivo conhecido por DAC (Dispositivo de Aplicação de Carga). Alguns trabalhos utilizam dispositivos especiais de aplicação de carga ${ }^{6} \mathrm{e}$ outros empregam máquinas de ensaio universal ${ }^{17,18}$. O presente estudo utilizou o dispositivo de aplicação de carga (DAC), de acordo com estudos relatados na literatura que utilizaram esse dispositivo para aplicação de cargas estáticas axiais e não axiais $^{10,11,13}$.

Quanto ao posicionamento linear dos extensômetros, este trabalho está de acordo com Heckmann et al. ${ }^{19}$, que estudaram deformações ao redor de implantes com estruturas metálicas 
de três elementos, e o trabalho de Nishioka et al. ${ }^{11}$. Este posicionamento justifica a pouca variação encontrada nas médias das deformações dos carregamentos axiais realizado pelo presente estudo, sendo que as maiores aferições foram encontradas nos pontos mais distantes do implante (D e E). Esta afirmação pode ser justificada com o trabalho de Çehreli, $\mathrm{Akça}^{6}$, que afirmaram que o posicionamento dos extensômetros tem influência nos resultados dos tipos de deformações encontradas.

A qualidade óssea é um fator de importância na eficácia do tratamento com implantes. O osso que os circunda não constitui um substrato homogêneo, de modo que suas propriedades físicas variam conforme a idade, o estado funcional e os fatores sistêmicos do paciente ${ }^{7}$. Diante disso, estudos in vitro têm empregado materiais considerados homogêneos e isotrópicos ${ }^{6,9,20}$. Associado a esses fatores, foi utilizado neste estudo um modelo homogêneo com propriedades elásticas uniformes, preconizadas em estudos que visam a simular o osso humano. Para isso, foi utilizado um bloco à base de poliuretano, que possui módulo de elasticidade semelhante ao tecido ósseo medular humano (Poliuretano: 3,6 GPa / osso medular: 4,0 a 4,5 GPa). Diversos fatores podem alterar os valores de microdeformações, como desenho das próteses e posicionamento dos extensômetros; porém, o padrão do uso do poliuretano como substrato ósseo permite uma melhor comparação entre os estudos. Assim, sugere-se que mais estudos sejam realizados para verificar as microdeformações em condições diferentes de configuração das próteses.

\section{CONCLUSÃO}

Dentro das limitações deste estudo, foi possível concluir que a localização da aplicação de carga influenciou a magnitude de microdeformação ao redor dos implantes. A aplicação de carga sobre os pontos não axiais $\mathrm{D}$ e E produziu um aumento da magnitude de microdeformação ao redor dos implantes.

\section{REFERÊNCIAS}

1. Adell R, Eriksson B, Lekholm U, Bränemark PI, Jemt T. A long-term follow-up study of osseointegrated implants in the treatment of totally edentulous jaws. Int J Oral Maxillofac Implants. 1990 Winter;5(4):347-59. PMid:2094653.

2. Lekholm U, Gröndahl K, Jemt T. Outcome of oral implant treatment in partially edentulous jaws followed 20 years in clinical function. Clin Implant Dent Relat Res. 2006; 8(4): 178-86. http://dx.doi.org/10.1111/j.1708-8208.2006.00019.x. PMid:17100743

3. Annibali S, Vestri AR, Pilotto A, La Monaca G, Di Carlo S, Cristalli MP. Patient satisfaction with oral implant rehabilitation: evaluation of responses to a questionnaire. Ann Stomatol (Roma). 2010 July; 1(3-4): 2-8. PMid:22238708.

4. Isidor F. Influence of forces on peri-implant bone. Clin Oral Implants Res. 2006 October; 17(S2, Suppl 2): 8-18. http://dx.doi.org/10.1111/j.16000501.2006.01360.x. PMid:16968378

5. Rangert BR, Sullivan RM, Jemt TM. Load factor control for implants in the posterior partially edentulous segment. Int J Oral Maxillofac Implants. 1997 May-June; 12(3): 360-70. PMid:9197101.

6. Çehreli MC, Akça K. Narrow-diameter implants as terminal support for occlusal three-unit FPDs: a biomechanical analysis. Int J Periodontics Restorative Dent. 2004 December; 24(6): 513-9. PMid:15626314.

7. Sahin S, Çehreli MC, Yalçin E. The influence of functional forces on the biomechanics of implant-supported prostheses-a review. J Dent. 2002 SeptemberNovember; 30(7-8): 271-82. http://dx.doi.org/10.1016/S0300-5712(02)00065-9. PMid:12554107

8. Wiskott HW, Belser UC. Lack of integration of smooth titanium surfaces: a working hypothesis based on strains generated in the surrounding bone. Clin Oral Implants Res. 1999 December; 10(6): 429-44. http://dx.doi.org/10.1034/j.1600-0501.1999.100601.x. PMid:10740452

9. Miyashiro M, Suedam V, Moretti Neto RT, Ferreira PM, Rubo JH. Validation of an experimental polyurethane model for biomechanical studies on implant supported prosthesis—-tension tests. J Appl Oral Sci. 2011 May-June; 19(3): 244-8. http://dx.doi.org/10.1590/S1678-77572011000300012. PMid:21625741

10. Abreu CW, Vasconcellos LGO, Balducci I, Nishioka RS. A comparative study of microstrain around three-morse taper implants with machined and plastic copings under axial loading. Braz J Oral Sci. 2010 January-March; 9(1): 11-5.

11. Nishioka RS, Vasconcellos LG, de Melo Nishioka GN. Comparative strain gauge analysis of external and internal hexagon, Morse taper, and influence of straight and offset implant configuration. Implant Dent. 2011 Apr;20(2):e24-32. http://dx.doi.org/10.1097/ID.0b013e318211fce8. PMid:21448016.

12. Vasconcellos LG, Nishioka RS, Vasconcellos LM, Nishioka LN. Effect of axial loads on implant supported partial fixed prostheses by strain gauge analysis. J Appl Oral Sci. 2011 Nov-Dec;19(6):610-5. PMid:22230995. PMCID: PMC3973462.

13. De Vasconcellos LG, Nishioka RS, De Vasconcellos LM, Balducci I, Kojima AN. Microstrain around dental implants supporting fixed partial prostheses under axial and non-axial loading conditions, in vitro strain gauge analysis. J Craniofac Surg. 2013 Nov;24(6):e546-51. http://dx.doi.org/10.1097/ SCS.0b013e31829ac83d. PMid:24220463.

14. Mericske-Stern R, Assal P, Merickse E, Bürgin W. Oclussal force and oral tactile sensibility measured in partially edentulous patients with ITI implants. Int J Oral Maxillofac Implants. 1995 May-Jun;10(3):345-53. PMid:7615331.

15. Jacques LB, Moura MS, Suedam V, Souza EA, Rubo JH. Effect of cantilever length and framework alloy on the stress distribution of mandibular-cantilevered implant-supported prostheses. Clin Oral Implants Res. 2009 Jul;20(7):737-41. http://dx.doi.org/10.1111/j.1600-0501.2009.01712.x. PMid:19489929.

16. Çehreli MC, Iplikçioglu H, Bilir OG. The influence of the location of load transfer on strains around implants supporting four unit cement-retained fixed prostheses: in vitro evaluation of axial versus off-set loading. J Oral Rehabil. 2002 Apr;29(4):394-400. PMid:11966975.

17. Karl M, Wichmann MG, Winter W, Graef F, Taylor TD, Heckmann SM. Influence of fixation mode and superstructure span upon strain development of implant fixed partial dentures. J Prosthodont. 2008 Jan;17(1):3-8. PMid:17927737. 
18. Suedam V, Souza EA, Moura MS, Jacques LB, Rubo JH. Effect of abutment's height and framework alloy on the load distribution of mandibular cantilevered implant-supported prosthesis. Clin Oral Implants Res. 2009 Feb;20(2):196-200. http://dx.doi.org/10.1111/j.1600-0501.2008.01609.x. PMid:19191796.

19. Heckmann SM, Karl M, Wichmann MG, Winter W, Graef F, Taylor TD. Cement fixation and screw retention: parameters of passive fit. An in vitro study of three-unit implant-supported fixed partial dentures. Clin Oral Implants Res. 2004 Aug;15(4):466-73. PMid:15248882.

20. Nishioka RS, Nishioka LN, Abreu CW, de Vasconcellos LG, Balducci I. Machined and plastic copings in three-element prostheses with different types of implant-abutment joints: a strain gauge comparative analysis. J Appl Oral Sci. 2010 May-Jun;18(3):225-30. PMid:20856998.

\section{CONFLITOS DE INTERESSE}

Os autores declaram não haver conflitos de interesse.

\section{*AUTOR PARA CORRESPONDÊNCIA}

Vinícius Anéas Rodrigues, Departamento de Materiais Dentários e Prótese, Instituto de Ciência e Tecnologia, UNESP - Univ Estadual Paulista, Av. Francisco José Longo, 777, 12245-000 São José dos Campos - SP, Brasil. e-mail: vinicius.rodrigues@fosjc. unesp.br

Recebido: Janeiro 24, 2014

Aprovado: Julho 16, 2014 